\title{
Quantifying oxygen distortions in lithium-rich transition-metal- oxide cathodes using ABF STEM
}

\author{
Emanuela Liberti*, Juan G. Lozano, Miguel Perez Osorio, Matthew R. Roberts, Peter G. \\ Bruce and Angus I. Kirkland
}

Department of Materials, University of Oxford, Parks Road, OX1 3PH, UK

\begin{abstract}
Lithium-rich cathodes can store excess charge beyond the transition metal redox capacity by participation of oxygen in reversible anionic redox reactions. Although these processes are crucial for achieving high energy densities, their structural origins are not yet fully understood. Here, we explore the use of annular bright-field (ABF) imaging in scanning transmission electron microscopy (STEM) to measure oxygen distortions in charged $\mathrm{Li}_{1.2 \mathrm{Ni}} \mathrm{NMn}_{2.6} \mathrm{O}_{2}$. We show that ABF STEM data can provide positional accuracies below $20 \mathrm{pm}$ but is restricted to cases where no specimen mistilt is present, and only for a range of thicknesses above $3.5 \mathrm{~nm}$. The reliability of these measurements is compromised even when the experimental and post-processing design are optimised for accuracy and precision, indicating that extreme care must be taken when attempting to quantify distortions in these materials.
\end{abstract}

\section{Introduction}

Advancements in the improvement of the energy density of lithium ion batteries is a major hurdle facing today's society. To date, efforts in the engineering optimisation of cells continue, however, substantial improvements via this approach are likely to be limited and thus new mechanisms of charge storage need to be identified. One approach is the use of lithium rich cathode materials which store charge beyond the normally accessible oxidation state changes of the transition metals and utilise anionic redox processes in the solid state [1-3]. These materials have the potential for cathodes with capacities in excess of $250 \mathrm{mAhg}^{-1}$. However, structural characterisation of the fully charged materials is essential to inform accurate theoretical models, for future materials discovery. In particular, since little is known about the local structure around oxidised oxygen, studies of the oxygen sublattice are of fundamental importance.

Techniques including X-ray diffraction (XRD) [4], and neutron diffraction (ND) [5] have been widely used to investigate structural transformations in working cathodes at high precision. However, these have limited site-specificity, and are unsuitable for studies of localised structural changes at the oxygen sites. In comparison, nuclear magnetic resonance (NMR) [3] and extended X-ray absorption fine structure (EXAFS) [6] use local probes to provide site-specific information but operate on relatively large length scales. Annular brightfield (ABF) imaging in scanning transmission electron microscopy (STEM) can directly visualize light and heavy elements [7], enabling both lithium and oxygen sites to be imaged together with transition metals at the atomic scale. A number of studies of lithium rich cathodes using ABF STEM can be found in the literature [8-13], however there are fewer attempts to directly quantify order-parameter fields such as distortions of oxygen column positions that provide new insights into the local structure of charged cathodes. In a recent study [13] ABF STEM has been used to measure local oxygen distortions following $\mathrm{O}^{2-}$ oxidation, in the $5 \mathrm{~d}$ 
transition metal system $\mathrm{Li}_{2} \mathrm{IrO}_{3}$. In this example, charge compensation by $\mathrm{O}^{2}$ oxidation results in the formation of peroxo-like species with shorter $\mathrm{O}-\mathrm{O}$ bond distances. However, in $5 \mathrm{~d}$ transition metal cathodes, anionic redox reactions in $3 \mathrm{~d}$ lithium rich transition metal oxides, such as $\mathrm{Li}_{1.2} \mathrm{Ni}_{0.2} \mathrm{Mn}_{0.6} \mathrm{O}_{2}$, do not form peroxo-like dimers but localised electron holes at the oxygen sites [1]. The structural rearrangement of the cathode in this case has been measured by several XRD [4], ND [5], NMR [3] and EXAFS [6] studies, but distortions in the oxygen sublattice have not been quantified..

In this study, we explore the possibility of using ABF STEM to measure lattice distortions of the oxygen framework in charged $\mathrm{Li}_{1.2} \mathrm{Ni}_{0.2} \mathrm{Mn}_{0.6} \mathrm{O}_{2}$. In particular, we investigate the robustness of this technique against possible errors in the measurements of oxygen atom column positions. We have combined established protocols in STEM post-processing to optimise the accuracy and precision in the determination of the oxygen peak locations and address current limitations of this technique in providing reliable measurements of distortions in these materials.

\section{Experiment and simulations}

\subsection{Synthesis}

Pristine $\mathrm{Li}\left[\mathrm{Li} 0.2 \mathrm{Ni}_{0.2} \mathrm{Mn}_{0.6}\right] \mathrm{O}_{2}$ was synthesized following a one-pot resorcinolformaldehyde method, as described elsewhere [14].

\subsection{ADF and ABF STEM}

Sample preparation of pristine material for STEM studies was carried out by crushing the as-synthesized powder in liquid nitrogen. Crushed particles were then dispersed in dipropyl carbonate (DPC) and the suspension was sonicated for $15 \mathrm{~min}$. The sample was then drop-cast onto a standard TEM grid coated with a holey carbon film. Charged samples were also crushed in liquid nitrogen in a glove box, under a controlled nitrogen atmosphere. In this case the TEM grids were loaded in a double tilt specimen holder, which was kept in a glove bag filled with nitrogen gas. The holder was then immediately transferred to the TEM column to minimise air exposure.

A probe-corrected cold field emission JEOL ARM200F operated at $200 \mathrm{kV}$ was used for STEM imaging. ABF images were acquired with a convergence semi-angle of $22 \mathrm{mrad}$ and inner and outer collection angles of $9.4-20.6 \mathrm{mrad}$ respectively. Multi-frame datasets were recorded with a probe current of $17.5 \mathrm{pA}$ and a total electron dose of $5 \times 10^{5} \mathrm{e}^{-/} \AA^{2}$ and $2 \times 10^{5}$ $\mathrm{e}^{-/} \AA^{2}$ for pristine and charged $\mathrm{Li}\left[\mathrm{Li}{ }_{0.2} \mathrm{Ni}_{0.2} \mathrm{Mn}_{0.6}\right] \mathrm{O}_{2}$, respectively (see subsequent text for details on the multi-frame approach).

All image data was acquired from specimens overhanging a hole in the grid, to avoid distortion artificats from the amorphous carbon support film.

\subsection{Image simulations}

Image simulations were performed using the multislice method [15-16] implemented using the MULTEM software [17]. Thermal vibrations were included using a frozen phonon approach [18-19] using an Einstein model with 30 phonon configurations. The Debye Waller factors of the elements were taken from previously reported data [20].

In all calculations a C2/m standard super cell was used [21]. The crystal was oriented along a [010] zone axis, with a size of $6.03 \mathrm{~nm} \times 5.95 \mathrm{~nm}(12 \times 12$ unit cells along the [001] and [100] axes). For the scanned region, a numerical real space grid of $86 \times 30$ scan positions 
was used with a sampling resolution of $0.16 \AA$ /pixel. A slice thickness of $2 \AA$ was used to slice the potential. To model specimen tilt, the super cell was tilted by $0.1^{\circ}$ around the $\mathbf{a}$ - and $\mathbf{b}$ - axes. All calculated images initially assumed an aberration-free probe. To account for residual probe aberrations a Gaussian broadening was applied, with a full width at half maximum (FWHM) of $0.6 \AA$. To determine peak positions from simulated images, a tessellation of 8 scan regions along the [100] axis was used. The main parameters used in the simulations are summarised in Table 1.

Table 1

Parameters of the multislice calculation.

\begin{tabular}{ll}
\hline Accelerating voltage & $200 \mathrm{kV}$ \\
Phonon configurations & 30 \\
Potential parameterization & Lobato et al. $[22]$ \\
Slice thickness & $0.2 \mathrm{~nm}$ \\
Probe convergence semi-angle & $22 \mathrm{mrad}$ \\
ABF inner and outer collection & $9.4-20.6 \mathrm{mrad}$ \\
angles & \\
Scan grid (reciprocal space) & $1024 \times 1024$ \\
Scan grid (real space) & $142 \times 49$ \\
Pixel size & $0.16 \AA$ \\
Gaussian broadening & $0.6 \AA$ \\
\hline
\end{tabular}

\subsection{Gaussian fitting}

To locate transition metal and oxygen peak positions, peak finding using Gaussian fitting was carried out, implemented in the StatSTEM software [23] (see subsequent text for further details). The width of the Gaussians was fixed for elements of the same type and the Gaussians were allowed to overlap to account for the presence of all elements.

\subsection{Density Functional Theory}

Details by Miguel 


\section{Results and discussion}

The load curve for $\mathrm{Li}\left[\mathrm{Li} 0.2 \mathrm{Ni}_{0.2} \mathrm{Mn}_{0.6}\right] \mathrm{O}_{2}$ is shown in Fig. 1 for the first cycle. The charge profile can be schematically divided into three regions corresponding to the main mechanisms of charge compensation. In the sloping region up to $4.4 \mathrm{~V}$, the capacity following $\mathrm{Li}^{+} \mathrm{de}-$ intercalation originates from the oxidation of $\mathrm{Ni}^{2+}$ to $\mathrm{Ni}^{4+}$. Further $\mathrm{Li}^{+}$extraction in the plateau region at $4.5 \mathrm{~V}$ activates anionic redox reactions [1-3] which are responsible for a significant improvement in capacity. Charging further to $4.8 \mathrm{~V}$ mainly results in oxygen loss. Diffusion mechanisms that govern oxygen mobility in the voltage plateau region at $4.5 \mathrm{~V}$ are currently the subject of intensive research and these mechanisms are likely to be driven by structural rearrangements of the oxygen sublattice. As an example, bond distortions effectively weaken the oxygen diffusion barrier, leading to an increase of the oxygen mobility NEED REF.

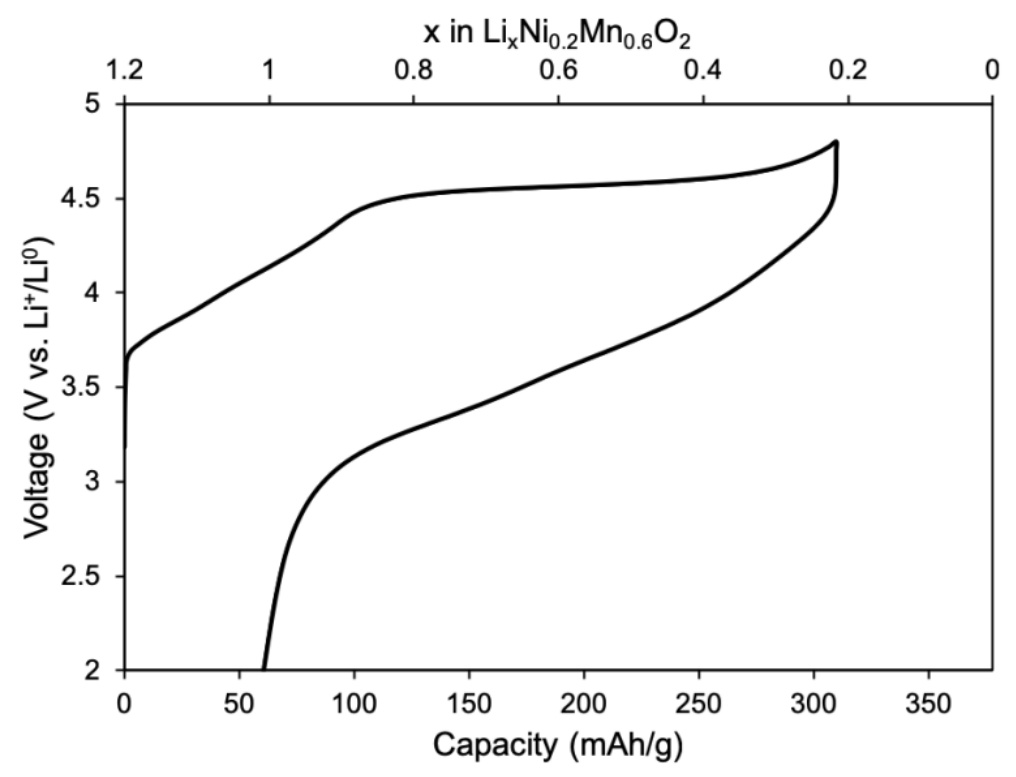

Figure 1. Load curve of $\mathrm{Li}\left[\mathrm{Li} 0.2 \mathrm{Ni}_{0.2} \mathrm{Mn}_{0.6}\right] \mathrm{O}_{2}$ for the first charge and discharge cycle. Three main regimes of charge compensation can be identified: Oxidation of the transition metal ions (slope region to $4.4 \mathrm{~V}$ ), anionic redox reactions (plateau at $4.5 \mathrm{~V}$ ) and oxygen loss (to $4.8 \mathrm{~V}$ ).

In this paper we report studies of structural distortions in the oxygen sublattice in the plateau region following charging of $\mathrm{Li}\left[\mathrm{Li} 0.2 \mathrm{Ni}_{0.2} \mathrm{Mn}_{0.6}\right] \mathrm{O}_{2}$ to $4.55 \mathrm{~V}$ using ABF STEM. Fig. 2 shows experimental images and structural models of the parent compound $\mathrm{Li}\left[\mathrm{Li}{ }_{0.2} \mathrm{Ni}_{0.2} \mathrm{Mn}_{0.6}\right] \mathrm{O}_{2}$, $\mathrm{Li}\left[\mathrm{Li}_{1 / 3} \mathrm{Mn}_{2 / 3}\right] \mathrm{O}_{2}$ in which $\mathrm{Ni}$ substitutes $\mathrm{Li}$ and $\mathrm{Mn}$, resulting in a layered $\mathrm{Li}\left[\mathrm{Ni}_{x} \mathrm{Li}_{1 / 3-2 \times / 3} \mathrm{Mn}_{2 / 3}-\right.$ $\mathrm{x} / 3] \mathrm{O}_{2}(\mathrm{x}=0.2)$ structure [24] before and after charge (Fig. $2 \mathrm{c}$ and $2 \mathrm{~d}$ respectively). In these images, features with the highest contrast indicate transition metal columns, while the faintest features correspond to Li positions between transition metal layers and intermediate contrast features are oxygen atom columns. The material is monoclinic in $\mathrm{C} 2 / \mathrm{m}$ and is viewed in a [010] projection (Fig. 2a). Upon initial removal of $\mathrm{Li}^{+}$during charging, it has been suggested that the $\mathrm{C} 2 / \mathrm{m}$ monoclinic symmetry transforms to $\mathrm{R} \overline{\mathrm{m}} \mathrm{m}$ symmetry by migration of the transition metal ions to the nearest vacant Li sites in the alkali metal layer [25] (Fig. 2b). In projection along a [010] zone axis, these two phases are indistinguishable, hence structural distortions are in principle, directly comparable independently of this phase transformation in the charged system. 
a

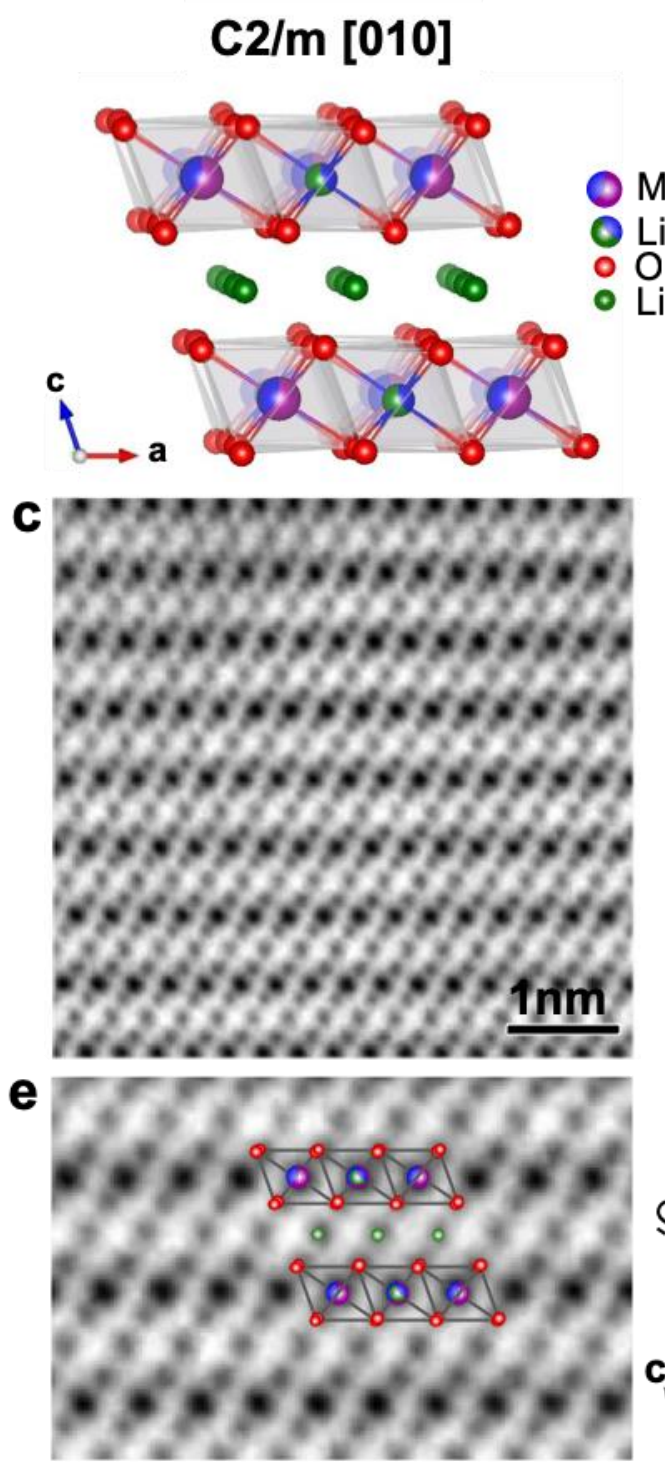

b

\section{$\mathbf{R} \overline{3} \mathbf{m}[010]$}

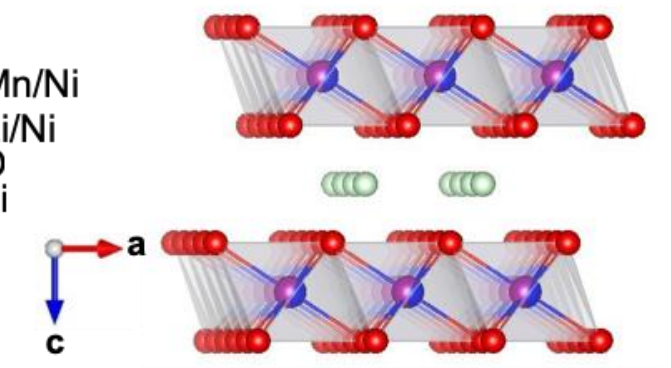

d
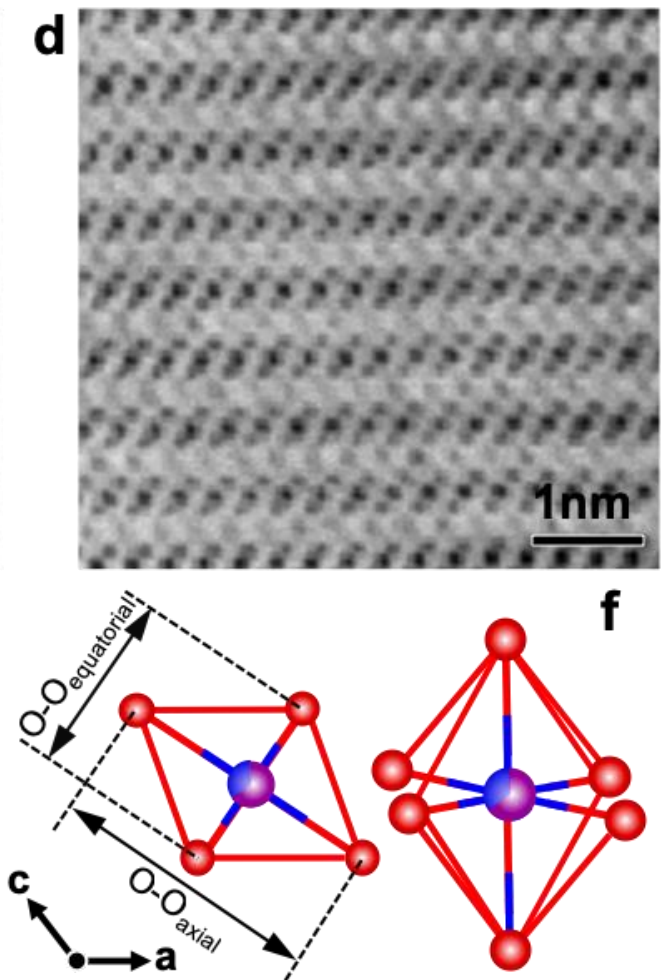

Figure 2. Atomic models of (a) monoclinic $C 2 / \mathrm{m}$ and (b) trigonal $\mathrm{R} \overline{3} \mathrm{~m}$ structures of $\mathrm{Li}\left[\mathrm{Li}{ }_{0.2} \mathrm{Ni}_{0.2} \mathrm{Mn}_{0.6}\right] \mathrm{O}_{2}$, oriented along [010]. In (b) light green atoms represent unoccupied $\mathrm{Li}$ sites, following delithiation. (c, d) Experimental ABF STEM images of pristine and charged $\mathrm{Li}\left[\mathrm{Li}{ }_{0.2} \mathrm{Ni}_{0.2} \mathrm{Mn}_{0.6}\right] \mathrm{O}_{2}$ respectively also oriented along [010]. (e) [010] projection of the atomistic model in (a) overlaid on an enlarged field of view taken from the experimental image in (c). (f) schematic of projected $\mathrm{O}-\mathrm{O}$ bond distances measured from $\mathrm{ABF}$ data, along the equatorial and axial planes of the $\mathrm{TmO}_{6}$ octahedra.

To predict the oxygen distortions that are expected in the oxygen sublattice, we have used $a b$ initio density functional theory (DFT), and calculated relaxed model structures of both pristine and charged $\mathrm{Li}\left[\mathrm{Li} 0.2 \mathrm{Ni}_{0.2} \mathrm{Mn}_{0.6}\right] \mathrm{O}_{2}$ assuming a $\mathrm{C} 2 / \mathrm{m}$ crystal symmetry. The expected changes in the projected distances are summarized in Table 2. For simplicity, we examined only the case of full delithiation at $4.8 \mathrm{~V}$, rather than the composition at $4.55 \mathrm{~V}$. In this case the projected $\mathrm{O}-\mathrm{O}$ bond distances, along the equatorial and axial planes of the $\mathrm{TmO}_{6}$ octahedra, contract by $20 \mathrm{pm}$ and $21 \mathrm{pm}$, respectively. The latter sets the upper bound for the accuracy required for measurement of the projected distances between oxygen columns in an ABF 
STEM image. However, this value falls below $5 \mathrm{pm}$ for projected cationic bond distances along the a- axis and charging to $4.4 \mathrm{~V}$ results in $<10 \mathrm{pm}$ change of the projected $\mathrm{O}-\mathrm{O}$ bond distances. The distortion observed in this voltage region results from the Jahn-Teller active $\mathrm{Ni}^{3+}$ site, which forms during oxidation of $\mathrm{Ni}^{2+}$ to $\mathrm{Ni}^{4+}$. The upper bound for the accuracy in this case is $6 \mathrm{pm}$, for the $\mathrm{O}-\mathrm{O}$ projections, which is considerably lower than for the case of charging to 4.8 V. As we will show later, measuring anionic projected distances with this level of precision is extremely challenging using ABF STEM data and thus in this work we have only investigated materials charged beyond $4.4 \mathrm{~V}$. Charging to higher voltages may lead to distortions that can be ascribed to oxygen activity although additional mechanisms of charge may also contribute to the overall observed structural changes.

\section{Table 2}

Projected atomic distances calculated from relaxed DFT model structures of pristine and charged $\mathrm{Li}\left[\mathrm{Li}{ }_{0.2} \mathrm{Ni}_{0.2} \mathrm{Mn}_{0.6}\right] \mathrm{O}_{2}$. The theoretical composition of the pristine material after relaxation is $\mathrm{Li}\left[\mathrm{Li}_{0.16} \mathrm{Ni}_{0.25} \mathrm{Mn}_{0.583}\right] \mathrm{O}_{2}$, while full delithiation is assumed for the charged material to $4.8 \mathrm{~V}$. The projected distances are averages of all the projected bond distances along the [010] orientation.

\begin{tabular}{lcccc}
\hline \multicolumn{1}{c}{ Structure } & $\begin{array}{c}\mathrm{O}-\mathrm{O}_{\text {equatorial }} \\
(\mathrm{pm})\end{array}$ & $\begin{array}{c}\mathrm{O}-\mathrm{O}_{\text {axial }} \\
(\mathrm{pm})\end{array}$ & $\begin{array}{c}\mathrm{Tm}-\mathrm{Tm}_{\mathrm{a}-} \\
(\mathrm{pm})\end{array}$ & $\begin{array}{c}\mathrm{Tm}-\mathrm{Tm}_{\mathrm{c}}- \\
(\mathrm{pm})\end{array}$ \\
\hline Pristine & 275 & 401 & 253 & 510 \\
Charged to 4.8 V & 255 & 380 & 248 & 532 \\
Distortion & -20 & -21 & -5 & +22 \\
Charged to 4.4 V & 269 & 391 & 250 & 516 \\
Distortion & -6 & -10 & -3 & +6 \\
\hline
\end{tabular}

The method we used to measure projected interatomic distances between oxygen and transition metal ion positions is based on statistical parameter estimation theory [26], and used the algorithm implemented in the StatSTEM software [23]. The intensities at the atomic column positions were fitted with a superimposition of Gaussian functions, to locate atomic sites both accurately and precisely. In ABF STEM the level of accuracy achievable by Gaussian fitting of image intensities is predominantly set by the amount of specimen mistilt, while the precision largely depends on the image signal to noise ratio and scan distortions. To minimize scan distortions that are typically present in single-frame STEM images, and to ensure that the precision of the measured atomic column positions is as high as possible, we used a multiframe serial acquisition to record the experimental images. In this method, a sequence of frames is acquired for mutually orthogonal scan directions and subsequently aligned using a non-rigid registration algorithm [27]. This experiment design reduces image artifacts such as sample/image drift, scan distortion and scan noise and significantly improves the precision in the location of peak positions [28-29], but also minimizes specimen damage by distributing the total electron dose across multiple frames. Gaussian fitting of ABF image intensities was therefore performed on averaged non-rigidly aligned ABF frames, which were processed using the Smart Align software [27]. Examples of averaged, non-rigidly aligned ABF frames are shown in Figs. 3a, 2b and 2c. Fig. 3b displays the fitted Gaussian model obtained from Fig. 3a, with peak positions shown in Fig. $3 \mathrm{c}$ for separate elements. 

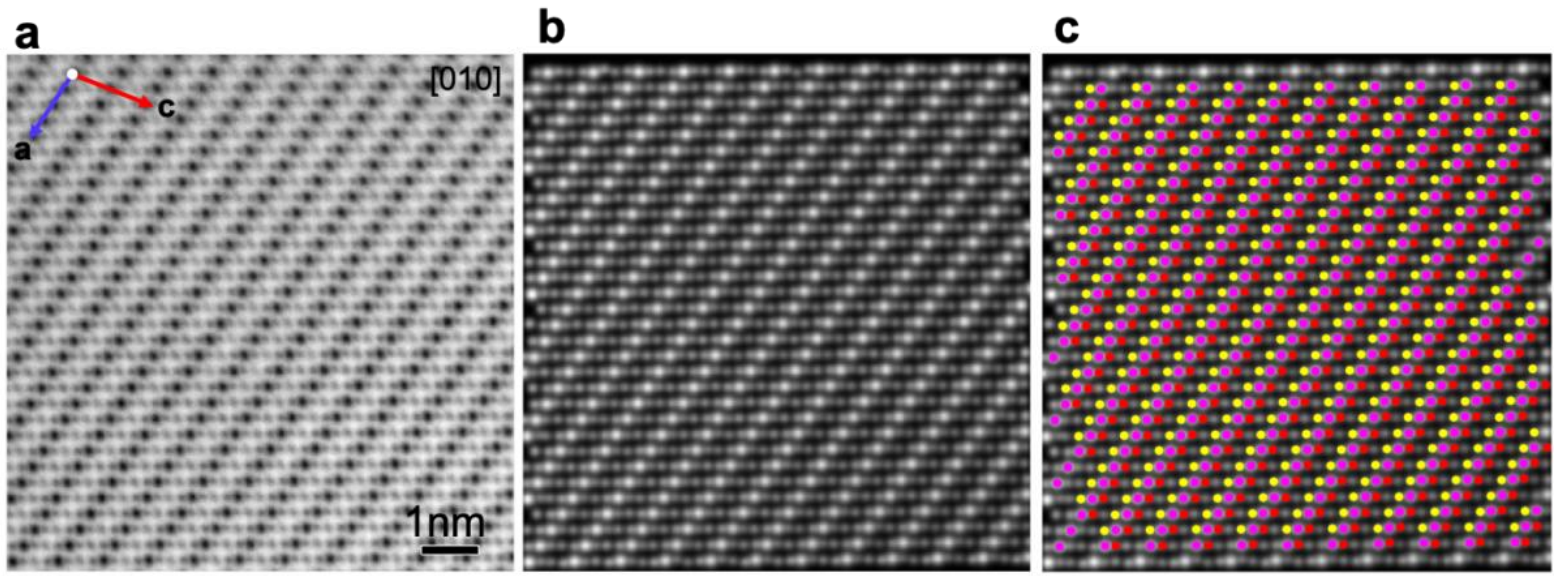

Figure 3. (a) Average ABF image of pristine $\mathrm{Li}\left[\mathrm{Li} 0.2 \mathrm{Ni}_{0.2} \mathrm{Mn}_{0.6}\right] \mathrm{O}_{2}$ obtained from a non rigid alignment of a data set of 32 images. Each frame was acquired with a dwell time of 3.8 us/pixel (512 x 512 pixels). The corresponding fitted Gaussian model is shown in (b). The fitting was carried out after inversion of the image contrast in (a). The background was set to 0 in vacuum regions. The estimated atomic columns positions are shown in (c) for oxygen (yellow and red) and the transition metal cations (magenta).

In addition to atom type and specimen thickness sample mistilt has been shown to affect the accuracy of interatomic distances measured from ABF STEM data [30]. ABF STEM images are particularly sensitive to small amounts of specimen tilt, in such a way that misalignments of few mrad can lead to unacceptably low accuracy when measuring anion positions. In addition, for specific elements and small mistilts the accuracy has been shown to vary non-linearly with thickness. This non-linearity in the error with thickness makes it difficult to predict the accuracy limit that can be achieved from ABF STEM frames if the specimen thickness is unknown. At high resolution, it is possible to measure thickness by modelmatching simulations and experiments, comparing the image contrast quantitatively [REF]. Quantitative approaches based on image simulations are challenging for the ABFmode, as modelling of the image formation process is complex, and can differ for heavy and light elements in a specimen [7]. An alternative method to determine specimen thickness is to count the number of atoms based on intensities in an ADF image, which is acquired simultaneously with the ABF. The counting method used in this approach is also based on statistical parameter estimation theory [31], and is implemented in the StatSTEM software [REF to StatSTEM]. In brief, the volume under the Gaussians used to estimate peak positions is treated as an integrated scattering cross-section, indicating the number of atoms that scatter along the beam direction. In the absence of detection noise, these scattering cross-sections are discrete components, each representing columns with the same number of atoms. However, in practice, the volumes are grouped into a probability distribution which is then decomposed into a discrete number of components according to an evaluation criterion which maximises the likelihood of the distribution. The robustness of any atom-counting method ultimately relies on the signal-tonoise ratio of the image intensities and is therefore generally applied to ADF images, where this is high for strongly scattering elements (e.g. the transition metals in this case). Furthermore, the assignment of a number of atoms to the cross-sections implies that integrated intensities must scale monotonically with thickness. This assumption is generally true for ADF, where the scattering is incoherent, but it is not necessarily satisfied by ABF, where image formation processes are partly coherent. However, unlike model-match simulation approaches, atom- 
counting methods based on scattering cross sections are robust against specimen mistilts and probe aberrations, and are therefore more reliable for thickness measurements.

Fig. 4 exemplifies how thickness can determined from an ADF image of pristine $\mathrm{Li}\left[\mathrm{Li}{ }_{0 .} \mathrm{Ni}_{0.2} \mathrm{Mn}_{0.6}\right] \mathrm{O}_{2}$. In this example, Fig. $4 \mathrm{a}$ is the averaged non-rigidly aligned ADF image (acquired simultaneously to the ABF data set) and Fig. 4b is the corresponding fitted model from which the atom-count was performed. Figs. $4 \mathrm{c}$ and $4 \mathrm{~d}$ show the histogram of the estimated scattering cross-sections and the resulting atom counts, respectively, ranging from a minimum of 12 transition metals to a maximum of 16 , corresponding to thicknesses of $3.5 \mathrm{~nm}$ and 4.6 $\mathrm{nm}$ respectively. It is worth noting that in the pristine specimen, $\mathrm{Ni}$ and $\mathrm{Mn}$ atom columns are mixed along the beam direction and as a consequence the scattering cross-sections vary according to the specific column composition, in addition to thickness.

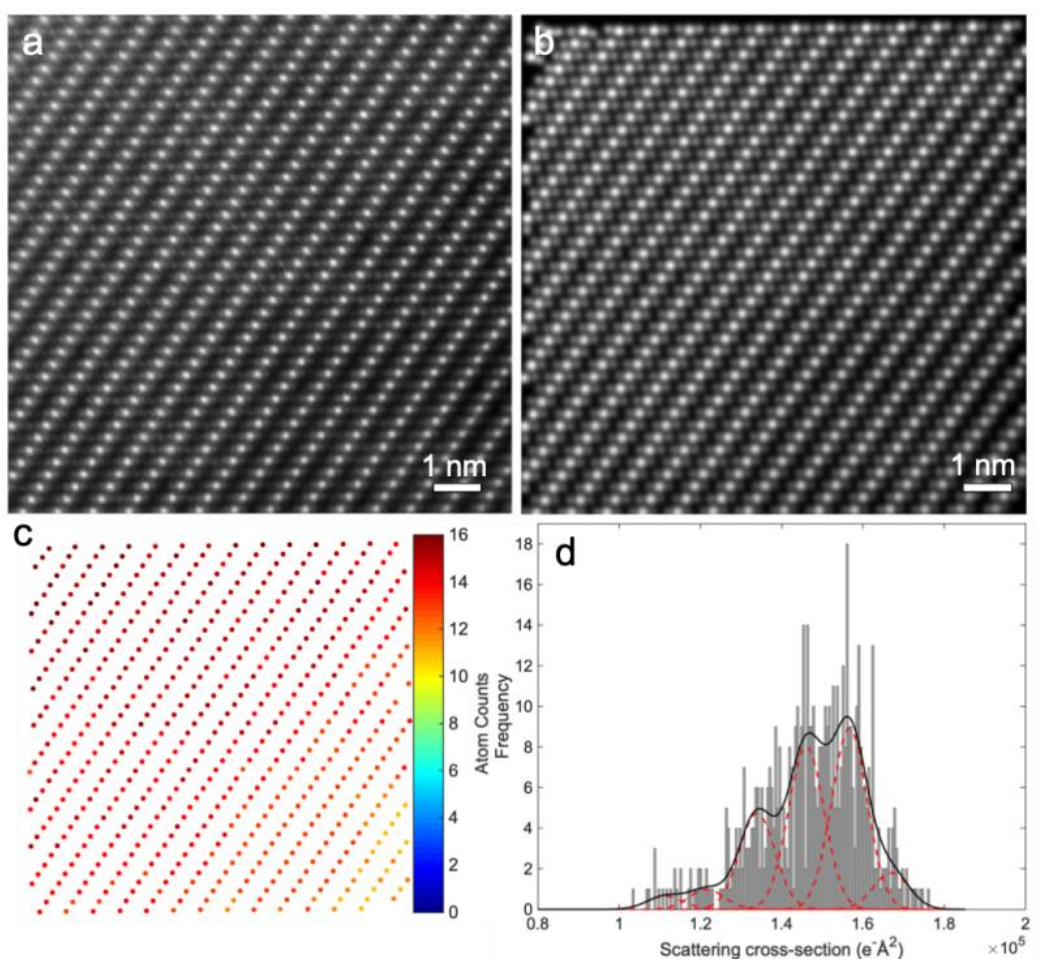

Figure 4. (a) Averaged non-rigidly aligned ADF image (acquired simultaneously to the ABF data set in Fig. 3a). (b) Corresponding fitted model on which the atom-count (c) was performed. (d) Histogram of the estimated scattering cross-sections, where individual components are highlighted in red.

Having determined the specimen thickness using atom-counting, the error in the measurement of the interatomic projected distances due to specimen mistilt can be estimated using simulations. The majority of the ABF data considered in this study suggest a specimen thickness ranging from 12 transition metals to 18, corresponding to specimen thicknesses of $3.5 \mathrm{~nm}$ and $5.2 \mathrm{~nm}$, respectively. We therefore performed ABF image simulations, using the multislice method [REFS] implemented in the MULTEM code [17] and analysed the effect of mistilt for thicknesses in this range only. The results are summarised in Tables 3, 4 and 5, for thicknesses of $3.5 \mathrm{~nm}, 4.4 \mathrm{~nm}$ and $5.2 \mathrm{~nm}$, respectively. Each table gives a comparison for a specimen on-axis versus the case of a misalignment of $0.1^{\circ}$ around the $\mathbf{a}$ - and $\mathbf{b}$ - directions. Mistilts larger than this value are unlikely, as the shortest $\mathrm{O}-\mathrm{O}$ projected atomic distance would no longer be resolvable experimentally. Following the simulations, we performed Gaussian 
fitting. The deviation in the tables is the difference between the calculated distances using a Gaussian fit and the reference value in the simulated model, while the error is the standard deviation of the measured distances. The model structure used in this case is that of the parent compound $\mathrm{Li}\left[\mathrm{Li}_{1 / 3} \mathrm{Mn}_{2 / 3}\right] \mathrm{O}_{2}$. This choice was justified by the computationally demanding task of constructing a supercell from ab initio calculations with realistic experimental thickness and composition.

Table 3. Comparison of mean projected $\mathrm{O}-\mathrm{O}$ and $\mathrm{TM}-\mathrm{TM}$ atomic columns distances from Gaussian fitting of simulated ABF STEM images at $0^{\circ}$ and $0.1^{\circ}$ specimen mistilt, for a specimen thickness of $3.5 \mathrm{~nm}$.

\begin{tabular}{ccccc}
\hline & $\begin{array}{c}\mathrm{O}-\mathrm{O}_{\text {equatorial }} \\
(\mathrm{pm})\end{array}$ & $\begin{array}{c}\mathrm{O}-\mathrm{O}_{\text {axial }} \\
(\mathrm{pm})\end{array}$ & $\begin{array}{c}\text { Tm-Tma- } \\
(\mathrm{pm})\end{array}$ & $\begin{array}{c}\text { Tm-Tmc- } \\
(\mathrm{pm})\end{array}$ \\
\hline Standard & 270 & 398 & 246 & 503 \\
\hline $0^{\circ}$ & 290.3 & 399.0 & 246.7 & 501.1 \\
Deviation & $20.3 \pm 1.4$ & $1 \pm 1.1$ & $0.7 \pm 1.4$ & $-1.9 \pm 0.2$ \\
\hline $0.1^{\circ}$ & 289.5 & 394.3 & 238.9 & 498.8 \\
Deviation & $19.5 \pm 4.0$ & $-3.7 \pm 4.8$ & $-7.1 \pm 5.1$ & $-4.2 \pm 0.9$ \\
\hline
\end{tabular}

Table 4. Comparison of mean projected $\mathrm{O}-\mathrm{O}$ and $\mathrm{Tm}-\mathrm{Tm}$ atomic columns distances from Gaussian fitting of simulated ABF STEM images at $0^{\circ}$ and $0.1^{\circ}$ specimen mistilt, for a specimen thickness of $4.4 \mathrm{~nm}$.

\begin{tabular}{ccccc}
\hline & $\begin{array}{c}\mathrm{O}-\mathrm{O}_{\text {equatorial }} \\
(\mathrm{pm})\end{array}$ & $\begin{array}{c}\mathrm{O}-\mathrm{O}_{\text {axial }} \\
(\mathrm{pm})\end{array}$ & $\begin{array}{c}\text { Tm-Tma- } \\
(\mathrm{pm})\end{array}$ & $\begin{array}{c}\text { Tm-Tmc- } \\
(\mathrm{pm})\end{array}$ \\
\hline Standard & 270 & 398 & 246 & 503 \\
\hline $0^{\circ}$ & 287.5 & 397.7 & 247.7 & 503.1 \\
Deviation & $17.5 \pm 1.6$ & $-0.3 \pm 0.3$ & $1.7 \pm 1.9$ & $-0.1 \pm 0.5$ \\
\hline $0.1^{\circ}$ & 284.5 & 391.5 & 239.8 & 500.6 \\
Deviation & $14.5 \pm 4.4$ & $-6.5 \pm 5.3$ & $-6.2 \pm 5.0$ & $-2.4 \pm 0.9$ \\
\hline
\end{tabular}

Table 5. Comparison of mean projected $\mathrm{O}-\mathrm{O}$ and $\mathrm{Tm}-\mathrm{Tm}$ atomic columns distances from Gaussian fitting of simulated ABF STEM images at $0^{\circ}$ and $0.1^{\circ}$ specimen mistilt, for a specimen thickness of $5.2 \mathrm{~nm}$.

\begin{tabular}{ccccc}
\hline & $\begin{array}{c}\mathrm{O}-\mathrm{O}_{\text {equatorial }} \\
(\mathrm{pm})\end{array}$ & $\begin{array}{c}\mathrm{O}-\mathrm{O} \text { axial } \\
(\mathrm{pm})\end{array}$ & $\begin{array}{c}\text { Tm-Tma- } \\
(\mathrm{pm})\end{array}$ & $\begin{array}{c}\text { Tm-Tmc- } \\
(\mathrm{pm})\end{array}$ \\
\hline Standard & 270 & 398 & 246 & 503 \\
\hline $0^{\circ}$ & 283.8 & 395.2 & 247.7 & 502.8 \\
Deviation & $13.8 \pm 0.4$ & $-2.8 \pm 0.7$ & $1.7 \pm 1.9$ & $-0.2 \pm 0.6$ \\
\hline $0.1^{\circ}$ & 281.9 & 389.4 & 239.7 & 500.9
\end{tabular}


Mistilt clearly affects the accuracy of the measured projected atomic distances, to the extent that deviations from the true values become larger than the upper bound for the accuracy set by the expected DFT distortion. However, if no mistilt is present, the accuracy remains below $20 \mathrm{pm}$ for $\mathrm{O}-\mathrm{O}$ interatomic distances for specimens of larger thicknesses, and below $5 \mathrm{pm}$ for the cations, indicating that oxygen sublattice distortions can only be reliably measured for thicknesses above $3.5 \mathrm{~nm}$. We also note that the inclusion of Poisson noise in the calculations will reduce the precision in the measured projected interatomic distances and may result in larger errors. However, for the fixed, total electron dose that is experimentally required to minimise specimen damage, the precision that can be achieved by minimising image distortions is the highest one attainable.

Having addressed possible sources of errors when measuring interatomic distances using ABF STEM, which mostly arise from specimen mistilt, scan distortions and the signalto-noise ratio, we now present experimental results obtained from the pristine specimen. The data are summarised in Fig. 5 for specimens of comparable thicknesses.
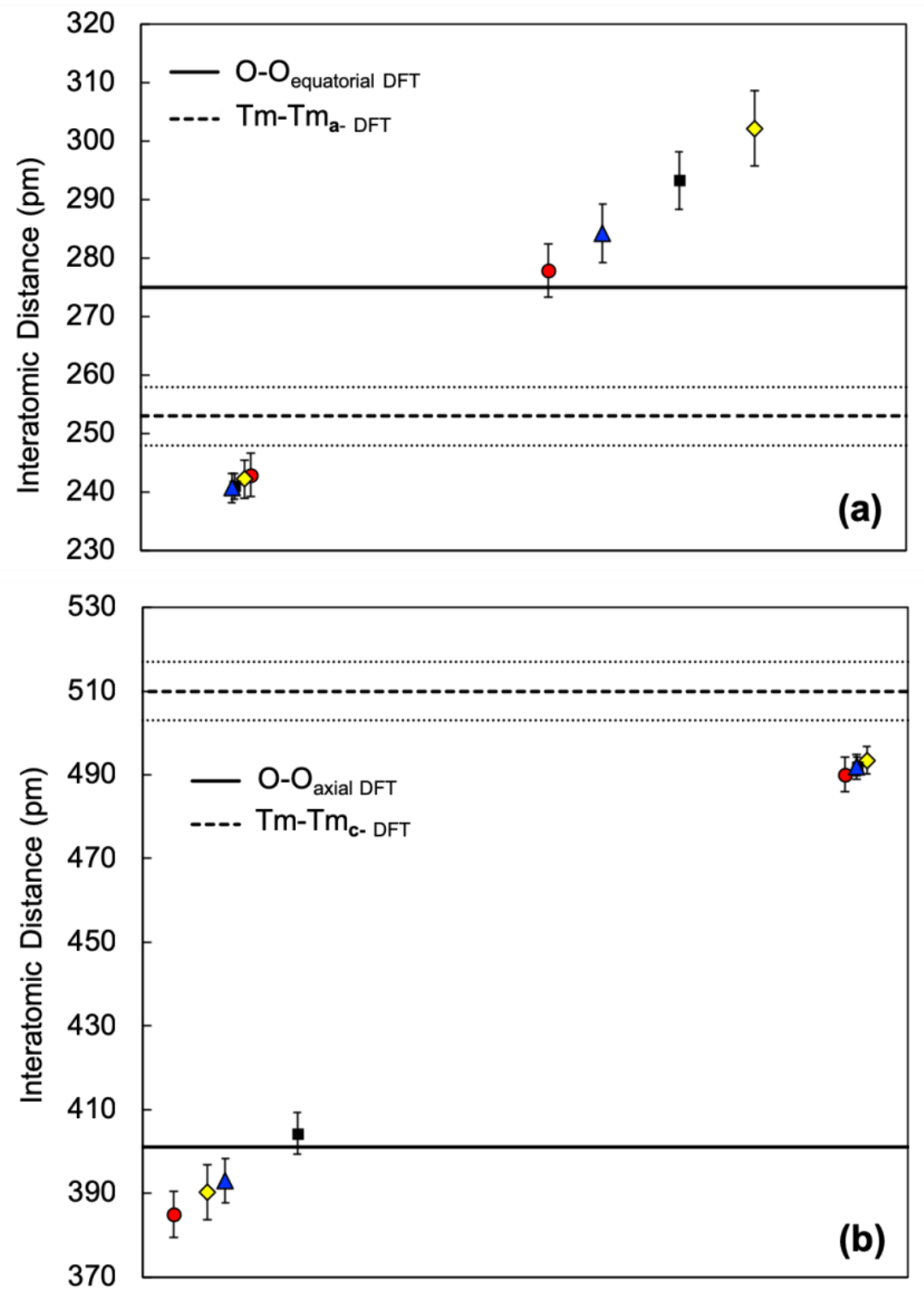
Figure 5. Summary of ABF STEM data sets of pristine $\mathrm{Li}\left[\mathrm{Li} 0.2 \mathrm{Ni}_{0.2} \mathrm{Mn}_{0.6}\right] \mathrm{O}_{2}$. (a) and (b) display mean projected $\mathrm{O}-\mathrm{O}$ and $\mathrm{Tm}-\mathrm{Tm}$ interatomic columns distances measured using Gaussian fitting.

We now exemplify how interatomic distances can vary significantly for different sets of measurements (markers of different shapes). The data points are plotted against DFT calculated reference values, indicated by solid or dashed lines for $\mathrm{O}-\mathrm{O}$ or Tm-Tm projected distances respectively. The deviation of the DFT calculated projected distances from refined neutron powder diffraction data are also shown in the figures, for the Tm-Tm projections only, as the $\mathrm{O}-\mathrm{O}$ projected distance deviation is only of order $1 \mathrm{pm}$. It is obvious that the accuracy worsens for the $\mathrm{O}-\mathrm{O}$ equatorial projections, where the oxygen columns are in close proximity to each other and the transition metals are located between the anions. A similar result has also been obtained by Finley and co-workers [7], who observed that the measurement of the intercolumn spacing between oxygen and heavy elements pairs may not be reliable using $\mathrm{ABF}$ data, even if the contrast at these sites is clearly resolvable. It is also worth noting that the data collected from the anions span a larger range of distances than the cations, where the reproducibility of the measurements is higher.

As the precision in peak position location is optimised via the experimental design, one obvious source of error is specimen mistilt. To determine if this is present in the experimental data, we fitted ADF maxima positions and confirmed that the interatomic distances between the cations matched those measured from the ABF images, and that the fitted minima in the $\mathrm{ABF}$ images were located at the same positions in the ADF images. An example is given in Table 6, for one of the data sets analysed.

Table 6. Comparison between mean projected $\mathrm{O}-\mathrm{O}$ and $\mathrm{Tm}-\mathrm{Tm}$ atom column distances from Gaussian fitting of ABF and ADF STEM peak intensities in pristine $\mathrm{Li}\left[\mathrm{Li} 0 .{ }_{2} \mathrm{Ni}_{0.2} \mathrm{Mn}_{0.6}\right] \mathrm{O}_{2}$ (averaged non-rigidly aligned data sets). The corresponding $\mathrm{ABF}$ averaged frame is shown in Fig. 3b.

\begin{tabular}{cccc}
\hline & $\begin{array}{c}\text { ABF } \\
(\mathrm{pm})\end{array}$ & $\begin{array}{c}\text { ADF } \\
(\mathrm{pm})\end{array}$ & $\begin{array}{c}\text { Deviation } \\
(\mathrm{pm})\end{array}$ \\
\hline $\mathrm{Tm}_{\mathrm{T}}-\mathrm{Tm}_{\mathbf{a}-}$ & $240.7 \pm 2.5$ & $240.9 \pm 2.6$ & $0.2 \pm 0.1$ \\
$\mathrm{Tm}-\mathrm{Tm}_{\mathbf{c}-}$ & $491.9 \pm 2.9$ & $492.3 \pm 2.2$ & $0.7 \pm 0.4$ \\
\hline
\end{tabular}

For all cases examined and shown in Fig. 5 we found similar values for the deviation between ABF and ADF Tm-Tm interatomic distances, indicating that a small amount of mistilt is present in the images. The irreproducibility of the anion measurements in the ABF must therefore be ascribed to additional factors affecting the accuracy of the data, such as the thickness variation across the particle (at 12 atom counts the accuracy is low), and also the complexity of this structure in projection.

Previous ADF STEM characterization studies of lithium rich transition metal oxide cathodes have shown that an intrinsic defect spinel/rock-salt layer grows on the surface of the specimen at selected facets of the crystal, with a specific orientation relationship (also shown in Fig. 6). As the STEM image contrast is dominated by the structure at the entrance surface of the sample, this thin layer of spinel/rock-salt will have a significant influence on the final image contrast. The experimental conditions used for imaging in this study $(200 \mathrm{kV}$ accelerating voltage, $22 \mathrm{mrad}$ probe convergence semi-angle) give a depth of field under optimised imaging 
conditions of $\Delta \mathrm{z} 8.4 \mathrm{~nm}$ [32]. This implies that the final projected structure recorded in the $\mathrm{ABF}$ images must be considered as an average over a significantly larger depth. Moreover, the contribution of the surface will be larger at the edge of the particles and will decrease towards the thicker regions as shown schematically in the inset in Fig. 6b. When the STEM probe is located at position A it propagates through only the surface layer and therefore the recorded image shows the projected structure of the rock-salt/spinel phase. When the electron probe is at position $\mathrm{B}$, the surface layer and the bulk structure contribute almost equally and the final image contrast is a superposition of the contrast from both phases. At position $\mathrm{C}$ the contribution of the surface layer to the final image contrast is negligible and the image shows the bulk structure only. In this work most ABF STEM data were recorded with the electron probe located at positions similar to B and hence the surface layer definitely contributes to the recorded image contrast. For this complex structure it is very challenging to estimate oxygen distortions using structures calculated ab initio which implies that the values in Table 1 may not be representative on a local scale, although they are representative over a larger scale. Furthermore, small misalignments between the surface phase and the bulk may also further limit the accuracy of the measured distortions.

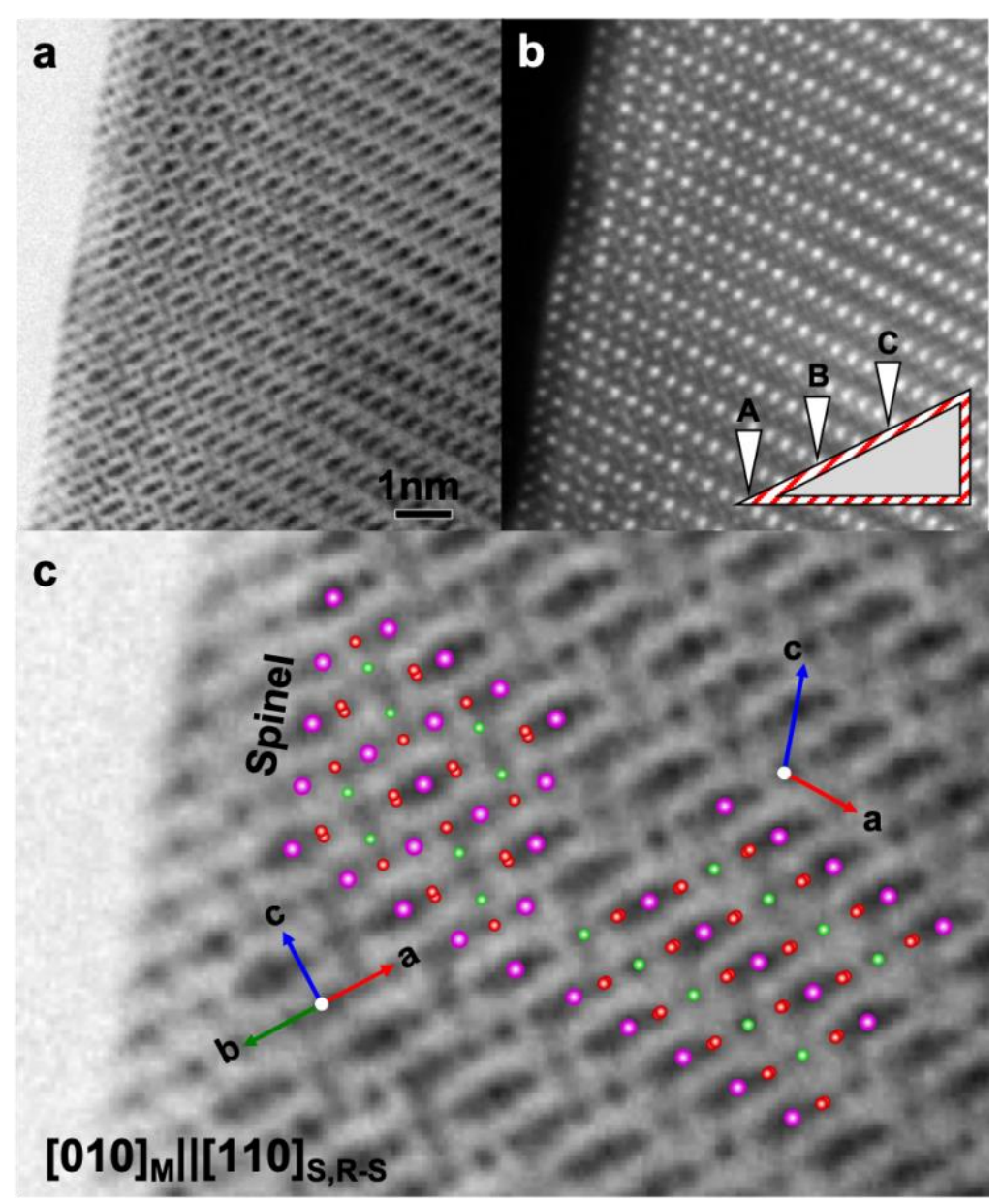

Figure 6: (a), (b) Average non-rigidly aligned ABF and ADF images of $\mathrm{Li}[\mathrm{Li} 0.2 \mathrm{Ni} 0.2 \mathrm{Mn} 0.6] \mathrm{O}_{2}$, acquired at the edge of the specimen, showing the spinel/rock-salt termination. In (b) a schematic representation of the effect of depth of focus is also shown. The contribution of the surface spinel phase to the final image contrast is more significant near the edge of the particle and decreases in the thicker regions where the bulk structure dominates. (c) an enlarged view 
taken from (a) illustrating the overlap between bulk and surface phases with orientation relationship [010]M $\|[110]_{\mathrm{S}, \mathrm{R}-\mathrm{S} \text {. }}$

In some cases, the accuracy of the oxygen interatomic distances remains below $20 \mathrm{pm}$, as shown in Table 7. Here we define the accuracy as the deviation of the mean measured distances from the true distance, referenced to the relaxed DFT model, and the precision as the standard deviation in the measured distances.

Table 7. Comparison between mean projected $\mathrm{O}-\mathrm{O}$ and $\mathrm{Tm}-\mathrm{Tm}$ interatomic distances in pristine $\mathrm{Li}\left[\mathrm{Li} 0.2 \mathrm{Ni}_{0.2} \mathrm{Mn}_{0.6}\right] \mathrm{O}_{2}$ measured from $\mathrm{ABF}$, DFT and neutron powder diffraction data [14].

\begin{tabular}{lccc}
\hline & $\begin{array}{c}\text { ABF } \\
(\mathrm{pm})\end{array}$ & $\begin{array}{c}\text { Deviation from DFT } \\
(\mathrm{pm})\end{array}$ & $\begin{array}{c}\text { Deviation from ND } \\
(\mathrm{pm})\end{array}$ \\
\hline O-Oequatorial & $284.3 \pm 5.0$ & $9.3 \pm 5.0$ & $10.3 \pm 5.0$ \\
O-O & $-8 \pm 5.2$ & $-7 \pm 5.2$ \\
$\mathrm{Tm}-\mathrm{Tm}_{\mathbf{a}-}$ & $2403.0 \pm 5.2$ & $-12.3 \pm 2.5$ & $-7.3 \pm 2.2$ \\
$\mathrm{Tm}-\mathrm{Tm}_{\mathbf{c}-}$ & $491.9 \pm 2.9$ & $-18.1 \pm 2.9$ & $-11.1 \pm 2.9$ \\
\hline
\end{tabular}

Taking these data as reference measurements for the pristine structure, we then compared these values against those in the charged material. Table 8 summarises the results for particles in the same thickness range.

Table 8. Measured distortion of projected $\mathrm{O}-\mathrm{O}$ and $\mathrm{Tm}-\mathrm{Tm}$ interatomic distances from ABF data of charged $\mathrm{Li}\left[\mathrm{Li}_{0.2} \mathrm{Ni}_{0.2} \mathrm{Mn}_{0.6}\right] \mathrm{O}_{2}$ versus the pristine material. Comparison with the expected deviation from DFT is also shown. For the charged material, we assumed a $\mathrm{C} 2 / \mathrm{m}$ reference lattice.

\begin{tabular}{lcc}
\hline & $\begin{array}{c}\text { Deviation from } \\
\text { pristine ABF } \\
(\mathrm{pm})\end{array}$ & $\begin{array}{c}\text { Deviation from } \\
\text { pristine DFT } \\
(\mathrm{pm})\end{array}$ \\
\hline $\mathrm{O}-\mathrm{O}_{\text {equatorial }}$ & $-14.05 \pm 4.15$ & -20 \\
$\mathrm{O}-\mathrm{O}_{\text {axial }}$ & $-6.2 \pm 3$ & -21 \\
$\mathrm{Tm}-\mathrm{Tm}_{\mathbf{a}-}$ & $+2.6 \pm 1.8$ & -5 \\
$\mathrm{Tm}-\mathrm{Tm}_{\mathbf{c}-}$ & $+12.5 \pm 1.4$ & +22 \\
\hline
\end{tabular}

Although in this particular case the distortions measured from ABF data follow the trend predicted by DFT, except for the smallest Tm-Tm distance along the a- axis which requires the smallest accuracy of $5 \mathrm{pm}$, the results were not reproducible for all charged particles examined. This indicates that it is generally challenging to determine if a meaningful experimental value for the anionic distortion can be measured from the data. As for the pristine material, the charged specimen also has an extremely complex structure which further complicates analysis of the ABF images.

Previous NMR studies on charged materials have shown that $\mathrm{Li}^{+}$is extracted simultaneously from both the alkali ion layers and the Li sites in the transition metal layers [3]. This is however in apparent disagreement with the features visible in the ABF images (e.g. Fig. 
2d), which shows that the Li sites in the alkali layers are not vacant following charging at 4.55 V. STEM studies [REF] have attributed the contrast in these layers to a surface migration of transition metal ions as a consequence of electron beam irradiation. However, the experiments reported here were conducted under carefully controlled dose conditions and thus we exclude that the contrast at the Li layers is associated with a change in occupancy due to electron beam damage during image acquisition. Instead, other mechanisms contribute to the contrast in this region. Firstly, the effect of the overlap between the defect spinel/rock-salt structure at the surface and the bulk structure persists in the charged material. Firstly this overlap may be intrinsic to the pristine particle, or forms during delithiation from a migration of transition metal ions into vacant octahedral Li sites between the transition metal layers in the near surface region. Secondly, not all lithium is extracted from the alkali layers on charging in the plateau region [24] and to a lesser extent, this residual Li also contributes to the contrast in the alkali Li layers. Thirdly, it has been suggested that migration of the transition metal ions during charge, not only occurs at the surface, leading to the spinel (or rock-salt) reconstruction, but may also occur in the bulk, following dynamic oxygen redox reactions [33]. This partially reversible bulk migration of the metals also contributes to the contrast in ABF data. Finally, it has been suggested that Ni may also be present at the surface in a reduced state, which may lead to an overall change in the local oxygen coordination, that is different to that predicted from DFT based on a simple fully delithiated model [33].

Distinguishing between the contributions of these different mechanisms to the image contrast is extremely challenging and partially explains the variations in measurements of local structural distortion from $\mathrm{ABF}$ images.

\section{Conclusions}

In this work we have explored the limitations of the ABF STEM technique for measuring oxygen distortions in $\mathrm{Li}\left[\mathrm{Li} 0 .{ }_{2} \mathrm{Ni} 0.2 \mathrm{Mn} 0.6\right] \mathrm{O}_{2}$ cathodes, following charging and $\mathrm{Li}^{+}$ deintercalation. The activity of the oxygen sublattice is known to play an important role in the charging mechanisms of these materials, however quantification of the required structural parameters at the atomic scale is challenging, and remains unreported to date. Here we have used ABF STEM to image oxygen sites alongside the lithium and the transition metal sites and have explored ways to quantify oxygen interatomic projected distances from image intensities of both pristine and charged specimens. For the pristine material a protocol that establishes the conditions under which an upper bound for the accuracy can be achieved is described. This bound is confirmed by DFT which predicts a distortion as low as $20 \mathrm{pm}$ for projected $\mathrm{O}-\mathrm{O}$ interatomic distances, if the material is fully delithiated. Our results show that this level of accuracy is very difficult to achieve, even when experimental and post-processing designs are optimised. In particular, small amounts of specimen mistilt significantly affect the measurements, but even when these are absent, the accuracy achievable is insufficient to detect structural distortions in the anion sublattice for sample thicknesses below $4 \mathrm{~nm}$. The reliability of ABF STEM is further compromised by the complex structure of these materials, especially for the charged case. 


\section{References}

[1].K. Luo, M. R. Roberts, R. Hao, N. Guerrini, D. M. Pickup, Y.-S. Liu, K. Edström, J. Guo, A. V. Chadwick, L. C. Duda, P. G. Bruce Charge-compensation in $3 d-$ transition-metal-oxide intercalation cathodes through the generation of localized electron holes on oxygen, Nature Chem. 8 (2016) 684-691.

[2]. K. Luo, M. R. Roberts, N. Guerrini, N. Tapia-Ruiz, R. Hao, F. Massel, D. M. Pickup, S. Ramos, Y.-S. Liu, J. Guo, A. V. Chadwick, L. C. Duda, P. G. Bruce, Anion Redox Chemistry in the cobalt free $3 \mathrm{~d}$ transition metal oxide intercalation electrode Li[Li0.2Ni0.2Mn0.6]O2, J. Am. Chem. Soc. 138 (2016) 11211-11218.

[3].A. R. Armstrong, M. Holzapfel, P. Novák, C. S. Johnson, S.-H. Kang, M. M. Thackeray, P. G. Bruce, Demonstrating Oxygen Loss and Associated Structural Reorganization in the Lithium Battery Cathode $\mathrm{Li}\left[\mathrm{Ni}{ }_{0.2} \mathrm{Li}_{0.2} \mathrm{Mn}_{0.6}\right] \mathrm{O}_{2}, J$. Am. Chem. Soc. 128 (2006) 8694-8698.

[4].C. R. Fell, D. Qian, K. J. Carroll, M. Chi, J. L. Jones, Y. S. Meng, Correlation between oxygen vacancy, microstrain, and cation distribution in lithium-excess layered oxides during the first electrochemical cycle, Chem. Mater. 25 (2013) 1621-1629.

[5].H. Liu, C. R. Fell, K. An, L. Cai, Y. S. Meng, In-situ neutron diffraction study of the $\mathrm{xLi}_{2} \mathrm{MnO}_{3}(1-\mathrm{x}) \mathrm{LiMnO}_{2}(\mathrm{x}=0,0.5 ; \mathrm{M}=\mathrm{Ni}, \mathrm{Mn}, \mathrm{Co})$ layered oxide compounds during electrochemical cycling, J. Power Sources 240 (2013) 772-778.

[6]. S. Muhammad, H. Kim, Y. Kim, D. Kim, J. H. Song, J. Yoon, J.-H. Park, S.-J. Ahn, S.-H. Kang, M. M. Thackeray, W.-S. Yoon, Evidence of reversible oxygen participation in anomalously high capacity Li- and Mn-rich cathodes for Li-ion batteries, Nano Energy 21 (2016) 172-184.

[7].S. D. Findlay, N. Shibata, H. Sawada, E. Okunishi, Y. Kondo, Y. Ikuhara, Dynamics of annular bright field imaging in scanning transmission electron microscopy, Ultramicroscopy 110 (2010) 903-923.

[8]. S. D. Findlay, N. R. Lugg, N. Shibata, L. J. Allen, Y. Ikuhara, Prospects for lithium imaging using annular bright field scanning transmission electron microscopy: a theoretical study, Ultramicroscopy 111 (2011) 1144-1154.

[9]. R. Huang, T. Hitosugi, S. D. Findlay, C. A. J. Fisher, Y. H. Ikuhara, Y. H. Moriwake, H. Oki, Y. Ikuhara, Real-time direct observation of $\mathrm{Li}$ in $\mathrm{LiCoO}_{2}$ cathode material, Appl. Phys. Lett. 98, (2011), 051913.

[10]. R. Huang, Y. Ikuhara, STEM characterization for lithium-ion battery cathode materials, Curr. Opin. Solid State Mater. Sci. 16 (2012) 31-38. 
[11]. L. Gu, D. Xiao, Y.-S. Hu, H. Li, Y. Ikuhara, Atomic-Scale Structure Evolution in a Quasi-Equilibrated Electrochemical Process of Electrode Materials for Rechargeable Batteries, Advanced Materials 27 (2015) 2134-2149.

[12]. H. Yu, Y.-G. So, A. Kuwabara, E. Tochigi, N. Shibata, T. Kudo, H. Zhou, Y. Ikuhara, Crystalline Grain Interior Configuration Affects Lithium Migration Kinetics in Li-Rich Layered Oxide, Nano Lett. 16 (2016) 2907-2915.

[13]. E. McCalla, A. M. Abakumov, M. Saubanère, D. Foix, E. J. Berg, G. Rousse, M.-L. Doublet, D. Gonbeau, P. Novák, G. Van Tendeloo, R. Dominko, J.-M. Tarascon, Visualization of O-O peroxo-like dimers in high-capacity layered oxides for Li-ion batteries, Science 350 (2015) 1516-1521.

[14]. K. Luo, M. R. Roberts, R. Hao, N. Guerrini, E. Liberti, C. S. Allen, A. I. Kirkland, P. G. Bruce, One-Pot synthesis of lithium-rich cathode material with hierarchical morphology, Nano Lett. 16 (2016) 7503-7508.

[15]. J. M. Cowley, A. F. Moodie, The scattering of electrons by atoms and crystals. I. A new theoretical approach, Acta Cryst. 10 (1957) 609-619.

[16]. P. Goodman, A. F Moodie, Numerical evaluation of N-beam wave functions in electron scattering by the multislice method, Acta Cryst. A30 (1974) 280-290.

[17]. I. Lobato, D. Van Dyck, MULTEM: A new multislice program to perform accurate and fast electron diffraction and imaging simulations using Graphics Processing Units with CUDA, Ultramicroscopy 156 (2015) 9-17.

[18]. R. F. Loane, P. Xu, J. Silcox, Thermal vibrations in convergent-beam electron diffraction, Acta Crystallogr. Sect. A 47 (1991) 276-278.

[19]. D. A. Muller, B. Edwards, E. J. Kirkland, J. Silcox, Simulation of thermal diffuse scattering including a detailed phonon dispersion curve, Ultramicroscopy 86 (2001) 371-380.

[20]. A. Boulineau, L. Croguennec, C. Delmas, F. Weill, Reinvestigation of $\mathrm{Li}_{2} \mathrm{MnO}_{3}$ structure: electron diffraction and high resolution TEM, Chem. Mater. 21 (2009) 4216-4222.

[21]. P. Villars, K. Cenzual, $\mathrm{Li}_{2} \mathrm{MnO}_{3}$ Crystal Structure: Datasheet from "PAULING FILE Multinaries Edition - 2012" in SpringerMaterials (http://materials.springer.com/isp/crystallographic/docs/sd_1210246).

[22]. I. Lobato, D. Van Dyck, An accurate parameterization for scattering factors, electron densities and electrostatic potentials for neutral atoms that obey all physical constraints, Acta Cryst. A 70 (2014) 636-649. 
[23]. A. De Backer, K. H. W. van den Bos, W. Van den Broek, J. Sijbers, S. Van Aert, StatSTEM: An efficient approach for accurate and precise model-based quantification of atomic resolution electron microscopy images, Ultramicroscopy 171 (2016) 104-116.

[24]. Z. Lu, L. Y. Beaulieu, R. A. Donaberger, C. L. Thomas, J. R. Dahn, Synthesis, structure, and Electrochemical Behaviour of $\mathrm{Li}\left[\mathrm{Ni}_{x} \mathrm{Li}_{1 / 3-2 \times / 3} \mathrm{Mn}_{2 / 3-x / 3}\right] \mathrm{O}_{2}$, Electrochem. Soc. 149 (2002) A778-A791.

[25]. J. Zheng, P. Xu, M. Gu, J. Xiao, N. D. Browning, P. Yan, C. Wang, J.-G. Zhang, Structural and chemical evolution of Li- and Mn-rich layered cathode material, Chem. Mater. 27 (2015) 1381-1390.

[26]. A. J. den Dekker, S. Van Aert, A. van den Bos, D. Van Dyck, Maximum likelihood estimation of structure parameters from high resolution electron microscopy images. Part I: A theoretical framework, Ultramicroscopy 104 (2005) 83-106.

[27]. L. Jones, H. Yang, T. J. Pennycook, M. S. J. Marshall, S. Van Aert, N. D. Browning, M. R. Castell, P. D. Nellist, Smart Align - a new tool for robust nonrigid registration of scanning microscope data, Adv. Struct. Chem. Imaging 1 (2015) $1-16$.

[28]. N. Gauquelin, K. H. W. van den Bos, A. Béché, F. F. Krause, I. Lobato, S. Lazar, A. Rosenauer, S. Van Aert, J. Verbeeck, Determining oxygen relaxations at the interface: A comparative study between transmission electron microscopy techniques, Ultramicroscopy 181 (2017) 178-190.

[29]. M. Nord, P. E. Vullum, I. MacLaren, T. Tybell, R. Holmestad, Atomap: a new software tool for the automated analysis of atomic resolution images using twodimensional Gaussian fitting, Adv. Struct. Chem. Imaging 3 (2017) 1-12.

[30]. D. Zhou, K. Müller-Caspary, W. Sigle, F. F. Krause, A. Rosenauer, P. A. van Aken, Sample tilt effects on atom column position determination in ABF-STEM imaging, Ultramicroscopy 160 (2016) 110-117.

[31]. S. Van Aert, A. De Backer, G. T. Martinez, B. Goris, S. Bals, G. Van Tendeloo, Procedure to count atoms with trustworthy single-atom sensitivity, Phys. Rev. B 87 (2013) 064107.

[32]. E. C. Cosgriff, P. D. Nellist, A Bloch wave analysis of optical sectioning in aberration-corrected STEM, Ultramicroscopy 107 (2007) 626-634.

[33]. W. E. Gent, K. Lim, Y. Liang, Q. Li, T. Barnes, S.-J. Ahn, K. H. Stone, M. McIntire, J. Hong, J. H. Song, Y. Li, A. Mehta, S. Ermon, T. Tyliszczak, D. Kilcoyne, D. Vine, J.-H. Park, S.-K. Doo, M. F. Toney, W. Yang, D. Prendergast, 
W. C. Chueh, Coupling between oxygen redox and cation migration explains unusual electrochemistry in lithium-rich layered oxides, Nat. Comm. 8(2017) 2091.

\section{Acknowledgments}

The authors acknowledge Dr. K. Luo for providing the specimen, and assisting with sample preparation. The authors also acknowledge the European Union under the Seventh Framework Programme under a contract for an Integrated Infrastructure Initiative Reference 312483ESTEEM2. Financial support from EPSRC (Platform Grant EP/K032518/1) is also acknowledged. 